\title{
Assessment of anterior and posterior corneal indices using two Scheimpflug analyzers
}

\section{Índices da córnea anterior e posterior com dois analisadores Scheimpflug}

\author{
Dadud Charbel Fahd ${ }^{1}$, Carole George Cherfan ${ }^{1}$, Claudia Radd², Marc Asouad ${ }^{2}$, Shady Tanus Awwad ${ }^{1}$
}

\begin{abstract}
Purpose: To assess the agreement between the elevation and curvature measurements of the anterior and posterior corneal surfaces obtained using the Galilei Dual Scheimpflug Analyzer and those obtained using the Pentacam single Scheimpflug system.

Methods: This prospective, noninterventional, diagnostic study was conducted at the Department of Ophthalmology at the American University of Beirut (Medical Center) and included 60 eyes of 60 consecutive patients. Measurements were obtained using 2 different Scheimpflug analyzers (Galilei and Pentacam). The best-fit sphere was set at $8 \mathrm{~mm}$ in both machines. Pachymetry (CCT), anterior elevation (AE) and posterior elevation (PE), and curvature were assessed. Pearson's correlation coefficients, comparison of means, and Bland-Altman plots were used to evaluate agreement between the 2 systems.

Results: The average CCT (at the corneal apex) was $533 \pm 35 \mu \mathrm{m}$ and $532 \pm 37 \mu \mathrm{m}$ $(p=0.980)$, the average central AE was $1.25 \pm 3.95 \mu \mathrm{m}$ and $2.29 \pm 5.28 \mu \mathrm{m}(p=0.964)$ and the average central PE was $4.19 \pm 8.18 \mu \mathrm{m}$ and $5.42 \pm 14.05(p=0.956) \mu \mathrm{m}$ with the Galilei and Pentacam, respectively.

Conclusion: The Scheimpflug analyzers correlated well in the assessment of pachymetry, elevation, and curvature.

Keywords: Cornea/pathology; Cornea/anatomy \& histology; Corneal topography; Corneal pachymetry; Photography/instrumentation
\end{abstract}

\section{RESUMO}

Objetivo: Avaliar a concordância entre as medidas de elevação e curvatura das superfícies anterior e posterior da córnea obtidos pelos analisadores de Scheimpflug Galilei e Pentacam.

Método: Estudo de teste diagnóstico, prospectivo, não-intervencional realizado no Departamento de Oftalmologia do Centro Médico da Universidade Americana de Beirute. Sessenta olhos de 60 pacientes consecutivos. As medições foram feitas usando dois analisadores Scheimpflug diferentes (Galilei e Pentacam). A esfera de melhor ajuste foi fixada em $8 \mathrm{~mm}$ para ambos equipamentos. Paquimetria (CCT), elevação anterior (AE) e elevação posterior (PE), e curvatura foram avaliados. Coeficientes de correlação de Pearson, comparação das médias, e gráficos de Bland-Altman foram utilizados para avaliar a correlação.

Resultados: A média de CCT (no ápice da córnea) foi $533 \pm 35$ um usando o Galilei e $532 \pm 37$ um usando o Pentacam $(p=0,980)$. As médias centrais de AE foram de 1,25 \pm 3,95 $\mu \mathrm{m}$ e 2,29 \pm e 5,28 $\mu \mathrm{m}$ com o Galilei e Pentacam, respectivamente $(p=0,964)$. As médias de centrais de PE foram 4,19 $\pm 8,18 \mu \mathrm{m}$ e 5,42 $\pm 14,05 \mu \mathrm{m}$ com a Galilei e Pentacam, respectivamente $(p=0,956)$.

Conclusões: Os analisadores de Scheimpflug avaliados correlacionam bem em termos de paquimetria, elevação e curvatura

Descritores: Córnea/patologia; Córnea/anatomia ehistologia; Topografia da córnea; paquimetria corneana; Fotografia/instrumentação.

\section{INTRODUCTION}

Corneal laser surgery is currently the most widely used surgical method for correcting refractive errors. Measurement of the corneal curvature, thickness, and elevation are important for the preoperative assessment of patients requiring refractive surgery and for the diagnosis of corneal ectasias like keratoconus and pellucid marginal degeneration. Historically, a simple Placido-based corneal topography was considered the norm for screening the cornea for topographic anomalies. Currently, Scheimpflug and Placido systems are being used to provide information on the anterior and posterior corneal surfaces. Currently, 2 such systems are widely used: the Galilei Dual Scheimpflug Analyzer (Ziemer, SIS, Port, Switzerland) and the Pentacam (OCULUS Optikgeräte GmbH, Germany) single Scheimpflug analyzer.

The present study aims to evaluate the agreement in measurements obtained by these 2 systems. Previous studies have evaluated the agreement between Scheimpflug devices; however, different best-fit sphere (BFS) diameters were used in both devices ${ }^{(1)}$ or only elevation data were compared between the Dual Scheimpflug Analyzer and a Scanning Slit-beam imaging system ${ }^{(2)}$. Comparisons using different BFS would alter the elevation data obtained by the single Scheimpflug system (compared with the dual Scheimpflug system) because the reference is flatter. Now that the pre-set BFS diameter in the single Scheimpflug system is universally set to $8.0 \mathrm{~mm}$, we attempted a comparison with equal BFS diameters in both systems. Our aim was not to demonstrate the superiority of one system over the other, but to discuss the similarities between both systems.

\section{METHODS}

This prospective, noninterventional, diagnostic study comprised 60 eyes of normal candidates for refractive surgery. Patients who had
Funding: No specific financial support was available for this study.

Disclosure of potential conflicts of interest: D.C. Fahd, None; C.G. Cherfan, None; C. Raad, None; M. Asouad, None; S.T. Awwad, None.

Correspondence address: Shady T. Awwad. Director of Laser and Refractive Surgery Division - Department of Ophthalmology - American University of Beirut Medical Centre, Beirut, Lebanon E-mail: sawwad@gmail.com 
undergone previous ocular surgeries, those with any systemic disease (including diabetes mellitus, connective tissue disease, hypertension, and collagen vascular diseases), and/or those who had worn rigid contact lenses in the past 6 months were excluded. Soft contact lens wearers were asked to discontinue wearing their contact lenses for at least 14 days prior to measurements ${ }^{(3)}$. The study received the approval of the Institutional Review Board of the American University of Beirut and complied with the Health Insurance Portability and Accountability Act (HIPAA) recommendations. Informed consent was obtained from all patients. All subjects underwent corneal topography using the Galilei (dual Scheimpflug system) followed by corneal topography using the Pentacam (single Scheimpflug system) by the same trained ophthalmic technician who was blinded to the study protocol.

\section{Pentacam (single Scheimpflug system)}

The Pentacam system uses a single rotating Scheimpflug camera $\left(180^{\circ}\right)$ and a monochromatic slit-light source [blue light-emitting diode (LED) at $475 \mathrm{~nm}$ ] that rotate together around the optical axis of the eye to calculate a 3-dimensional (3D) model of the anterior segment. The 3D, high-resolution, cornea-scanning mode obtains 50 images of the eye in 1 second. Overall, 138,000 true elevation points were recorded.

\section{Galilei (dual Scheimpflug system)}

The Galilei Dual Scheimpflug Analyzer uses two rotating Scheimpflug cameras integrated with a Placido topographer. The light source is also monochromatic (blue LED at $475 \mathrm{~nm}$ ). The 122,000 data points are analyzed per scan. The system used Scheimpflug images of all anterior segment structures (cornea, iris, pupil, anterior chamber, and lens) to evaluate and analyze the corneal shape and thickness, pupil size, and anterior chamber parameters (size, volume, and angle). The presence of two Scheimpflug camera devices that obtain images of the same part of the eye simultaneously decreases movement artifacts and increases image accuracy by superimposing the two images. The presence of the Placido improves the accuracy of calculation of the anterior corneal curvature.

\section{Measurement technioue}

Measurements were obtained in a semi-lit room. To standardize the tear film meniscus and account for tear film abnormalities that may influence Placido measurements, a drop of carboxymethyl cellulose artificial tears was instilled in the fornix of each eye, and measurements were obtained 3 min later to ensure that the tear film meniscus was back to its normal thickness ${ }^{(4)}$. Both eyes were measured. Measurements were obtained using an 8.0-mm diameter BFS, which was fitted in float. Central elevations and maximum/minimum elevations in the central $8 \mathrm{~mm}$ of the cornea were recorded with both the dual and single Scheimpflug systems. Pachymetry measurements were internally calculated by the individual machines by subtracting the anterior and posterior corneal elevations. In patients with a normal topography, only one eye was considered for analysis (60 eyes of 60 patients). Data from the right eyes of the first, third, and fifth 10 patients and data from the left eyes of the second, fourth, and sixth 10 patients were used for further analysis.

\section{Intraclass Correlation Coefficients (ICCs)}

For each system and for a defined subset of apparently healthy eyes, measurements were obtained 3 times and the data were averaged. One eye of each subject was selected. Ten right eyes were selected from the first 10 subjects and 10 left eyes were chosen from the second 10 subjects. The internal repeatability of each machine was analyzed by calculating the ICC, which was defined as the ratio of the between-subjects variance to the sum of the pooled within-subject variance and between-subjects variance. The ICC, which approached
1.0 when there was no variance between repeated measurements, was automatically calculated using PASW Statistics software (SPSS version 18.0, SPSS Inc. Chicago, IL, USA). The ICCs ranged from 0 to 1 and were commonly classified as follows: ICC $<0.75$, poor agreement; $0.75-<0.90$, moderate agreement; and $>0.90$, high agreement ${ }^{(5)}$

\section{Statistical analysis}

Statistical analysis was performed using the Statistical Program for Social Sciences v18.0 (SPSS v18.0, SPSS Inc. Chicago, IL, USA). A paired $t$-test was used to compare the instruments in each elevation parameter. Mean algebraic and mean absolute differences were calculated. Results are expressed as means \pm standard deviations. In addition, Pearson's correlation coefficients (PCCs) were calculated to assess the correlation between the anatomical parameters measured by each imaging technique. All tests were 2 -tailed. A $p$-value of $<0.05$ was considered statistically significant. Bland-Altman plots were used to assess the interchangeability (agreement) of the 2 imaging systems for measuring elevation parameters. These plots show the differences between the methods plotted against the mean of the methods. They provided a graphical method to assess the presence of an agreement between the 2 clinical techniques.

\section{RESULTS}

\section{Patient characteristics}

Sixty eyes of 60 subjects ( 25 males, 35 females) were included in the study. The mean age of the participants was $27.0 \pm 4.1$ years. All eyes had a normal topography, with no evidence of curvature abnormalities.

\section{MaCHINE CHARACTERISTICS}

Each measurement was obtained 3 times, and only the findings with the highest quality (as determined by the machine) were included for analysis. The ICC was calculated for each machine on the basis of 3 measurements. The ICC for the dual Scheimpflug system was as follows: 0.999 for pachymetry (CCT), 0.998 for SimK, 0.998 for anterior BFS, and 0.993 for posterior BFS. The ICC for the single Scheimplug system was as follows: 0.994 for CCT, 0.986 for SimK, 0.996 for anterior BFS, and 0.991 for posterior BFS.

\section{Pachymetry}

Pachymetry measurements are summarized in table 1. The mean difference between the dual Scheimpflug and single Scheimpflug systems in central pachymetry measurements was $0.7 \pm 8.2 \mu \mathrm{m}$. The mean absolute difference between the two machines in central pachymetry measurements was $6.7 \pm 4.6 \mu \mathrm{m}$. Figure 1 shows a Bland-Altman plot of central pachymetry. The dual Scheimpflug system exhibited a

Table 1. Correlation of pachymetry measurements between the dual Scheimpflug system (Galilei) and the single Scheimpflug system (Pentacam)

\begin{tabular}{lccc}
\hline $\boldsymbol{( \mu \mathrm { m } )}$ & $\begin{array}{c}\text { Galilei } \\
(\boldsymbol{\mu \mathrm { m }})\end{array}$ & $\begin{array}{c}\text { Pentacam } \\
(\boldsymbol{\mu \mathbf { m } )}\end{array}$ & $\begin{array}{c}\text { Pearson's correlation } \\
\text { coefficient }\end{array}$ \\
\hline Central pachymetry & $535 \pm 35$ & $534 \pm 37$ & 0.977 \\
Thinnest point & $531 \pm 37$ & $528 \pm 43$ & 0.982 \\
Superior 2 mm & $574 \pm 33$ & $590 \pm 33$ & 0.864 \\
Inferior 2 mm & $568 \pm 38$ & $559 \pm 43$ & 0.949 \\
Nasal 2 mm & $578 \pm 32$ & $579 \pm 34$ & 0.897 \\
Temporal 2 mm & $549 \pm 33$ & $553 \pm 34$ & 0.904 \\
Superior 3 mm & $612 \pm 35$ & $649 \pm 37$ & 0.889 \\
Inferior 3 mm & $613 \pm 35$ & $613 \pm 39$ & 0.865 \\
\hline
\end{tabular}


trend for displaying values larger than those displayed by the single Scheimpflug system for corneal thicknesses below $550 \mu \mathrm{m}$. It also exhibited a tendency to display smaller measurements at higher mean central pachymetry measurements. The PCC for central corneal thickness was 0.977 .

\section{Anterior and posterior elevation}

The anterior elevation measurements recorded by the 2 machines are summarized in table 2. Posterior elevation measurements recorded by the 2 machines are summarized in Table 3 . The mean difference in between the dual and single Scheimpflug systems in central AE and central PE measurements was $-1.04 \pm 2.48 \mu \mathrm{m}$ and $-1.38 \pm 7.08 \mu \mathrm{m}$, respectively. The mean absolute difference between the dual and single Scheimpflug systems in central AE and central PE measurements was $1.85 \pm 1.86 \mu \mathrm{m}$ and $2.77 \pm 6.10 \mu \mathrm{m}$, respectively. Anterior and posterior Bland-Altman plots of central elevation are shown in figure 2. A similar trend was observed for pachymetry measurements, with the dual Scheimpflug system showing higher and lower values than the single Scheimpflug system for lower and higher elevations, respectively. The PCC for central anterior elevation was 0.952, while that for central posterior elevation was 0.974 .

\section{Keratometric values}

Keratometric measurements recorded by the two machines are summarized in table 4 . The mean difference in anterior radii between the dual and single Scheimpflug systems in steep and flat meridians were $-0.05 \mathrm{~mm}$ and $-0.02 \mathrm{~mm}$, respectively. The mean absolute difference in anterior steep and flat meridian radii between the dual and

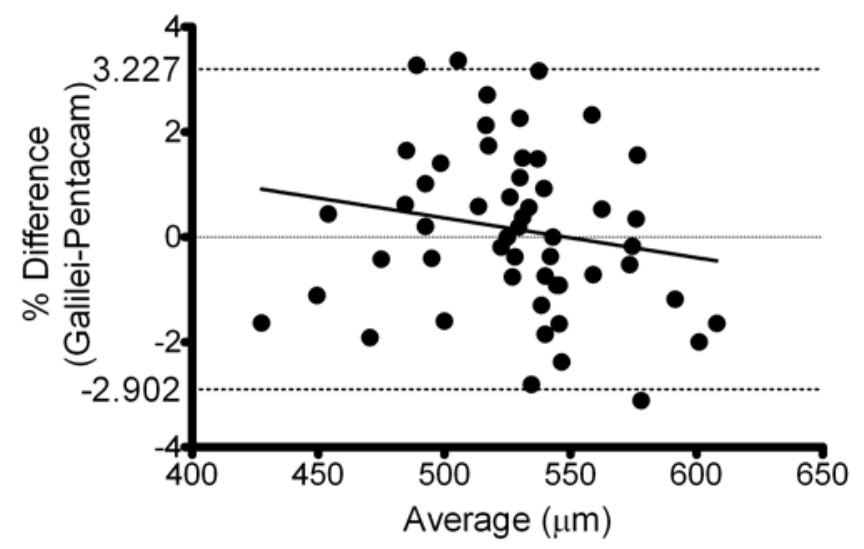

Figure 1. A Bland-Altman plot of percentage differences in average central pachymetry (CCT) measurements between the dual Scheimpflug system (Galilei) and the single Scheimpflug system (Pentacam).

Table 2. Correlation of central anterior elevation measurements between the dual Scheimpflug system (Galilei) and the single Scheimpflug system (Pentacam)

\begin{tabular}{lccc}
\hline $\mathbf{( \mu m )}$ & Galilei & Pentacam & $\begin{array}{c}\text { Pearson's correlation } \\
\text { coefficient }\end{array}$ \\
\hline Central elevation & $2.48 \pm 2.98$ & $4.23 \pm 4.57$ & 0.952 \\
Superior 2 mm & $-8.05 \pm 10.43$ & $-8.25 \pm 12.79$ & 0.933 \\
Inferior 2 mm & $-1.10 \pm 6.06$ & $1.35 \pm 12.81$ & 0.871 \\
Nasal 2 mm & $0.35 \pm 4.14$ & $-0.55 \pm 5.01$ & 0.784 \\
Temporal 2 mm & $1.30 \pm 4.37$ & $1.68 \pm 4.49$ & 0.749 \\
Superior 3 mm & $-8.43 \pm 8.73$ & $-12.08 \pm 10.33$ & 0.742 \\
Inferior 3 mm & $-10.18 \pm 9.89$ & $-9.30 \pm 7.11$ & 0.685 \\
\hline
\end{tabular}

single Scheimpflug systems was $0.08 \pm 0.14 \mathrm{~mm}$ and $0.06 \pm 0.07 \mu \mathrm{m}$, respectively. The mean difference in posterior steep and flat meridian radii between the dual and single Scheimpflug systems was $-0.02 \mathrm{~mm}$ and $-0.06 \mathrm{~mm}$, respectively. The mean absolute difference in the posterior steep and flat meridian radii between the dual and single Scheimpflug systems was $0.08 \pm 0.08 \mathrm{~mm}$ and $0.11 \pm 0.12 \mu \mathrm{m}$, respectively. The PCC for central average keratometry was 0.931

\section{DISCUSSION}

Our results demonstrated that both the Galilei Dual Scheimpflug Analyzer and the Pentacam Single Scheimpflug Analyzer display similar values for the same subjects measured in terms of keratometric readings, anterior and posterior elevations, and pachymetry. Both machines gave values comparable with the points lying along the line of equality, with small limits of agreements (Figures 1 and 2). Previous studies have reported good correlations between both machines in either pachymetry or corneal power measurements ${ }^{(6-8)}$

One of the main diagnostic tools for keratoconus and keratectasia is abnormal topography. Threshold values of topography, beyond which the topography would be labeled as abnormal, are specific for

Table 3. Correlation of central posterior elevation measurements between the dual Scheimpflug system (Galilei) and the single Scheimpflug system (Pentacam)

\begin{tabular}{lrcc}
\hline $\mathbf{( \mu m )}$ & Galilei & Pentacam & $\begin{array}{c}\text { Pearson's correlation } \\
\text { coefficient }\end{array}$ \\
\hline Central elevation & $5.33 \pm 7.69$ & $7.15 \pm 14.75$ & 0.974 \\
Superior 2 mm & $-20.43 \pm 16.49$ & $-22.63 \pm 24.59$ & 0.961 \\
Inferior 2 mm & $-3.25 \pm 11.79$ & $2.33 \pm 26.92$ & 0.807 \\
Nasal 2 mm & $1.23 \pm 10.84$ & $2.73 \pm 11.64$ & 0.804 \\
Temporal 2 mm & $4.50 \pm 11.86$ & $5.25 \pm 12.27$ & 0.961 \\
Superior 3 mm & $-22.65 \pm 10.75$ & $-35.03 \pm 17.11$ & 0.706 \\
Inferior 3 mm & $-24.95 \pm 17.77$ & $-26.53 \pm 18.26$ & 0.963 \\
\hline
\end{tabular}
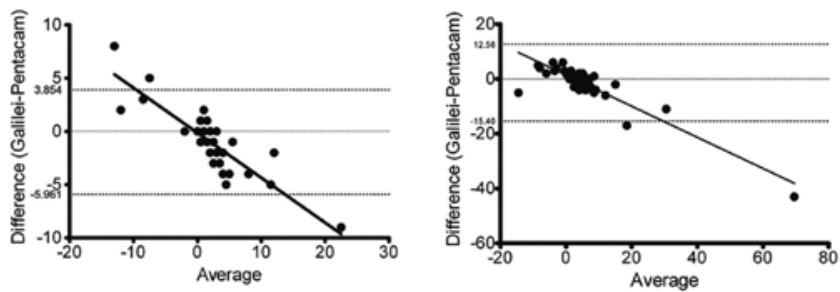

Figure 2. A Bland-Altman plot of differences in average central anterior elevation (AE) (left) and central posterior elevation (PE) (right) measurements between the dual Scheimpflug system (Galilei) and the single Scheimpflug system (Pentacam).

Table 4. Correlation of corneal keratometric measurements between the dual Scheimpflug system (Galilei) and the single Scheimpflug system (Pentacam)

\begin{tabular}{lccc}
\hline & Galilei & Pentacam & $\begin{array}{c}\text { Pearson's correlation } \\
\text { coefficient }\end{array}$ \\
\hline $\begin{array}{l}\text { Central average } \\
\text { keratometry (D) }\end{array}$ & $45.14 \pm 3.20$ & $44.81 \pm 3.02$ & 0.931 \\
R steep front $(\mathrm{mm})$ & $7.43 \pm 0.46$ & $7.46 \pm 0.44$ & 0.979 \\
R flat front $(\mathrm{mm})$ & $7.66 \pm 0.42$ & $7.68 \pm 0.40$ & 0.984 \\
R steep back $(\mathrm{mm})$ & $6.02 \pm 0.47$ & $6.03 \pm 0.48$ & 0.968 \\
R flat back $(\mathrm{mm})$ & $6.36 \pm 0.45$ & $6.42 \pm 0.40$ & 0.946 \\
\hline
\end{tabular}


each machine. Problems with the existence of 2 systems will make it more difficult to set common standard cut-off values. In addition, refractive surgery physicians who have access to both systems may be faced with the need to compare serial images of the same patient using 2 different machines. Machine threshold values were first studied on the Orbscan (Bausch and Lomb) $(2,9-11)$ and later on the Pentacam ${ }^{(12,13)}$. With the availability of the Galilei Dual Scheimpflug Analyzer, the primary concern was whether values obtained using the dual Scheimpflug system correlated with those obtained using the single Scheimpflug system and whether images captured by both systems can be safely compared. Our study showed that the 2 systems were strongly correlated in terms of keratometry, anterior and posterior elevation, and pachymetry measurements. Therefore, a conclusion or a trend in values in a specific patient can be generalized from the single Scheimpflug system and applied to the dual Scheimpflug system when considering threshold values and overall patterns. Our results, in terms of pachymetry, were in agreement with those published by Jahadi-Hoseini et al.(6).

The central keratometric, curvature, and pachymetric values showed a high correlation (and high agreement) and were not very different between both systems. As we moved peripherally, the correlation remained high, albeit not as strong as that in the central area. Similarly, other authors have reported an increase in variability in corneal pachymetry measurements from the center to the periphery, particularly in the superior cornea $a^{(4,14-17)}$. They attributed this variability to the effects of the superior eyelid ${ }^{(4)}$. We believe that the differences observed in paracentral (peripheral) values were very predictable because small decentration in the BFS would lead to a false high value, considering that the peripheral cornea is farther away from the BFS because of its prolate shape. Even within the same system, small decentrations in BFS can manifest as large deviations in peripheral elevation. The effects of contact lenses on corneal elevation and curvature have been eliminated from the equation because all subjects were advised to stop wearing soft contact lenses for at least 2 weeks ${ }^{(3)}$; furthermore, all subjects that had worn rigid contact lenses in the past 6 months were excluded from the study. The images were obtained on the same day, thus decreasing any additional confounding factors.

Keratometric values in both machines, despite the fact that they were obtained in a different manner, showed a high degree of correlation between both systems; keratometric values with the dual Scheimpflug system were obtained mainly from the Placido image and were only complemented by the rotating Scheimpflug cameras, while keratometry using the single Scheimpflug system relied solely on information from the Scheimpflug images.

The objective of our paper was not to show the advantage of one system over the other, but to assess the degree of correlation. A previously published study showed a difference between the single and dual Scheimpflug systems, with values obtained by the former system (Pentacam) being higher than those obtained by the latter (Galilei) $)^{(1)}$. We believe that the difference was primarily due to the different BFS diameters pre-set for each system: $9 \mathrm{~mm}$ in the single Scheimpflug system and $8 \mathrm{~mm}$ in the dual Scheimpflug system. Current versions of the Pentacam software, similar to the software used in our study, have adopted the 8-mm pre-set BFS diameter. The fact that both machines showed similar results for all indices made it possible to compare the follow-up findings of patients obtained with these two systems, provided the radius of the BFS was set to $8 \mathrm{~mm}$ in both machines.
The reason for incorporating two Scheimpflug cameras may solely be patency issues. The strong correlation between measurements obtained by both systems does not preclude the potential advantages offered by the dual Scheimpflug system over the single Scheimpflug system; in theory, the former should be less sensitive to eye movements while obtaining pachymetry measurements. This theoretical advantage could be translated into a clinical one only in a small subset of patients with eye movements beyond a certain critical threshold. Therefore, potential advantages of the dual Scheimpflug system observed in some patients would be easily diluted while evaluating aggregate results. Further studies with the objective of testing the theoretical advantages of the dual Scheimpflug system and comparing them with those of a gold standard system are required.

Our study was limited by the small sample size.

In conclusion, the single and dual Scheimpflug analyzers correlated well in terms of pachymetry, anterior and posterior elevation, and curvature measurements.

\section{REFERENCES}

1. Salouti R, Nowroozzadeh MH, Zamani M, Fard AH, Niknam S. Comparison of anterior and posterior elevation map measurements between 2 Scheimpflug imaging systems. J Cataract Refract Surg. 2009;35(5):856-62.

2. Sy ME, Ramirez-Miranda A, Zarei-Ghanavati S, Engle J, Danesh J, Hamilton DR. Comparison of posterior corneal imaging before and after LASIK using dual rotating scheimpflug and scanning slit-beam corneal tomography systems. J Refract Surg. 2013;29(2):96-101.

3. Hashemi H, Firoozabadi MR, Mehravaran S, Gorouhi F. Corneal stability after discontinued soft contact lens wear. Cont Lens Anterior Eye. 2008;31(3):122-5.

4. Miranda MA, Radhakrishnan H, O'Donnell C. Repeatability of oculus pentacam metrics derived from corneal topography. Cornea. 2009;28(6):657-66.

5. McGraw KO, Wong S.P. Forming inferences about some intraclass correlation coefficients. Psychological Methods. 1996;1:30-46.

6. Jahadi Hosseini HR, Katbab A, Khalili MR, Abtahi MB. Comparison of corneal thickness measurements using Galilei, HR Pentacam, and ultrasound. Cornea. 2010;29(10): 1091-5.

7. Oliveira CM, Ribeiro C, Franco S. Corneal imaging with slit-scanning and Scheimpflug imaging techniques. Clin Exp Optom. 2011;94(1):33-42.

8. Shirayama M, Wang L, Weikert MP, Koch DD. Comparison of corneal powers obtained from 4 different devices. Am J Ophthalmol. 2009;148(4):528-35 e1.

9. Cairns G, McGhee CN. Orbscan computerized topography: attributes, applications, and limitations. J Cataract Refract Surg. 2005;31(1):205-20

10. Rainer G, Findl O, Petternel V, Kiss B, Drexler W, Skorpik C, et al. Central corneal thickness measurements with partial coherence interferometry, ultrasound, and the Orbscan system. Ophthalmology. 2004;111(5):875-9.

11. Karimian F, Feizi S, Doozandeh A, Faramarzi A, Yaseri M. Comparison of corneal tomography measurements using Galilei, Orbscan II, and Placido disk-based topographer systems. J Refract Surg. 2011;27(7):502-8.

12. Lackner B, Schmidinger G, Pieh S, Funovics MA, Skorpik C. Repeatability and reproducibility of central corneal thickness measurement with Pentacam, Orbscan, and ultrasound. Optom Vis Sci. 2005;82(10):892-9.

13. O'Donnell C, Maldonado-Codina C. Agreement and repeatability of central thickness measurement in normal corneas using ultrasound pachymetry and the OCULUS Pentacam. Cornea. 2005;24(8):920-4.

14. Miranda MA, Radhakrishnan H, O'Donnell C. Repeatability of corneal thickness measured using an Oculus Pentacam. Optom Vis Sci. 2009;86(3):266-72.

15. Bullimore MA, Fusaro RE, Adams CW. The repeatability of automated and clinician refraction. Optom Vis Sci. 1998;75(8):617-22.

16. Lam AK, Chen D. Pentacam pachometry: comparison with non-contact specular microscopy on the central cornea and inter-session repeatability on the peripheral cornea. Clin Exp Optom. 2007;90(2):108-14.

17. Buehl W, Stojanac D, Sacu S, Drexler W, Findl O. Comparison of three methods of measuring corneal thickness and anterior chamber depth. Am J Ophthalmol. 2006; 141(1):7-12. 